\title{
Perioperative impact of sleep apnea in a high-volume specialty practice with a strong focus on regional anesthesia: a database analysis
}

\author{
Lukas Pichler, ${ }^{1,2}$ Sarah M Weinstein, ${ }^{1}$ Crispiana Cozowicz, ${ }^{1,2}$ Jashvant Poeran, ${ }^{3}$ \\ Jiabin Liu, ${ }^{1}$ Lazaros A Poultsides, ${ }^{4}$ Jawad N Saleh, ${ }^{1}$ Stavros G Memtsoudis, ${ }^{1,5}$
}

'Department of Anesthesiology,

Critical Care and Pain

Management, Hospital for

Special Surgery, New York, USA

2Paracelsus Medical University, Salzburg, Austria

${ }^{3}$ Icahn School of Medicine at Mount Sinai, New York, USA

${ }^{4}$ New York Langone Orthopaedic Hospital, New York, USA

5Weill Cornell Medical College, New York, USA

Correspondence to Stavros G Memtsoudis, Hospital for Special Surgery, New York 10021, USA:

memtsoudiss@hss.edu

Received 27 July 2018 Accepted 4 August 2018 Published Online First 11 January 2019
ABSTRACT

Background and objectives Obstructive sleep apnea (OSA) is a risk factor for adverse postoperative outcome and perioperative professional societies recommend the use of regional anesthesia to minimize perioperative detriment. We studied the impact of OSA on postoperative complications in a high-volume orthopedic surgery practice, with a strong focus on regional anesthesia.

Methods After Institutional Review Board approval, 41766 cases of primary total hip and knee arthroplasties (THAs/TKAs) from 2005 to 2014 were extracted from institutional data of the Hospital for Special Surgery (approximately 5000 THAs and 5000 TKAs annually, of which around $90 \%$ under neuraxial anesthesia).

The main effect was OSA (identified by the International Classification of Diseases, ninth revision codes); outcomes of interest were cardiac, pulmonary, gastrointestinal, renal/genitourinary, thromboembolic complications, delirium, and prolonged length of stay (LOS). Multivariable logistic regression models provided ORs, corresponding $95 \% \mathrm{Cls}$, and $\mathrm{p}$ values.

Results Overall, OSA was seen in $6.3 \%(n=1332)$ of patients with THA and $9.1 \%(n=1896)$ of patients with TKA. After adjustment for relevant covariates, OSA was significantly associated with $87 \%$ (OR 1.87, 95\% Cl 1.51 to 2.30$), 52 \%$ (OR $1.52,95 \% \mathrm{Cl} 1.13$ to 2.04$)$, and $44 \%$ (OR $1.44,95 \%$ Cl 1.31 to 1.57 ) increased odds for pulmonary gastrointestinal complications, and prolonged LOS, respectively. The odds for other outcomes remained unaltered by OSA diagnosis.

Conclusion We showed that, even in a setting with almost universal regional anesthesia use, OSA was associated with increased odds for prolonged LOS, and pulmonary and gastrointestinal complications. This puts forward the question of how effective regional anesthesia is in mitigating postoperative complications in patients with OSA.

\section{INTRODUCTION}

Obstructive sleep apnea (OSA) is a condition characterized by repetitive episodes of upper airway obstruction accompanied by cessation in airflow and intermittent hypoxemia. While many cases are undiagnosed, the prevalence of OSA is estimated to be as high as $10 \%$ to $30 \%$ in surgical patients, especially among those undergoing orthopedic surgery. ${ }^{1-3}$ Particularly troublesome is the association between OSA and increased risk of adverse postoperative outcomes. ${ }^{2}{ }^{4}$ These include pulmonary complications, postoperative cardiac events, and transfer to an intensive care unit as well as prolonged length of stay (LOS). ${ }^{56}$ Guidelines issued by perioperative professional societies recommend the use of regional anesthesia to reduce postoperative complications in patients with OSA, ${ }^{7-9}$ and studies have suggested improved postoperative outcomes with this approach in this patient population. ${ }^{10-12}$ Although lower joint arthroplasties are uniquely amenable to the use of neuraxia anesthesia, the majority of patients with OSA on a national level undergo this procedure under general anesthesia. $^{10}$

To date, no data are available on outcomes among patients with OSA in a practice that focuses on regional anesthesia as a routine anesthetic for total joint arthroplasty. We therefore studied the outcomes associated with total hip arthroplasty (THA) and total knee arthroplasty (TKA) procedures in a high-volume orthopedic specialty practice with a strong focus on regional anesthesia. We hypothesized that under these circumstances, OSA may still be associated with an increased risk of adverse postoperative outcomes, but to a limited extent.

\section{METHODS}

This is a retrospective cohort study using data from the Hospital for Special Surgery. Approximately 5000 THAs and 5000 TKAs are performed at this institution annually with around 90\% regional anesthesia use.

\section{Data sources and cohort}

Hospital billing datasets were used to extract patient characteristics and medical history which contained information such as age, sex, dates of admission and discharge, and International Classification of Diseases, ninth revision (ICD-9) codes.

For the period of 2005 to 2014, we identified 56 351 cases with procedure codes for THA (81.51) and TKA (81.54). Exclusion criteria were missing type of anesthesia $(n=10138)$, pediatric patients $(n=41)$, not the first procedure for the patient within the study time frame $(n=4145$; patients who had more than one surgery were included only once using data of their first visit), and surgeries that did not take place on the date of admission $(n=261)$. The final study cohort consisted of 41766 unique patients (21 022 THA; 20744 TKA)(figure 1). 


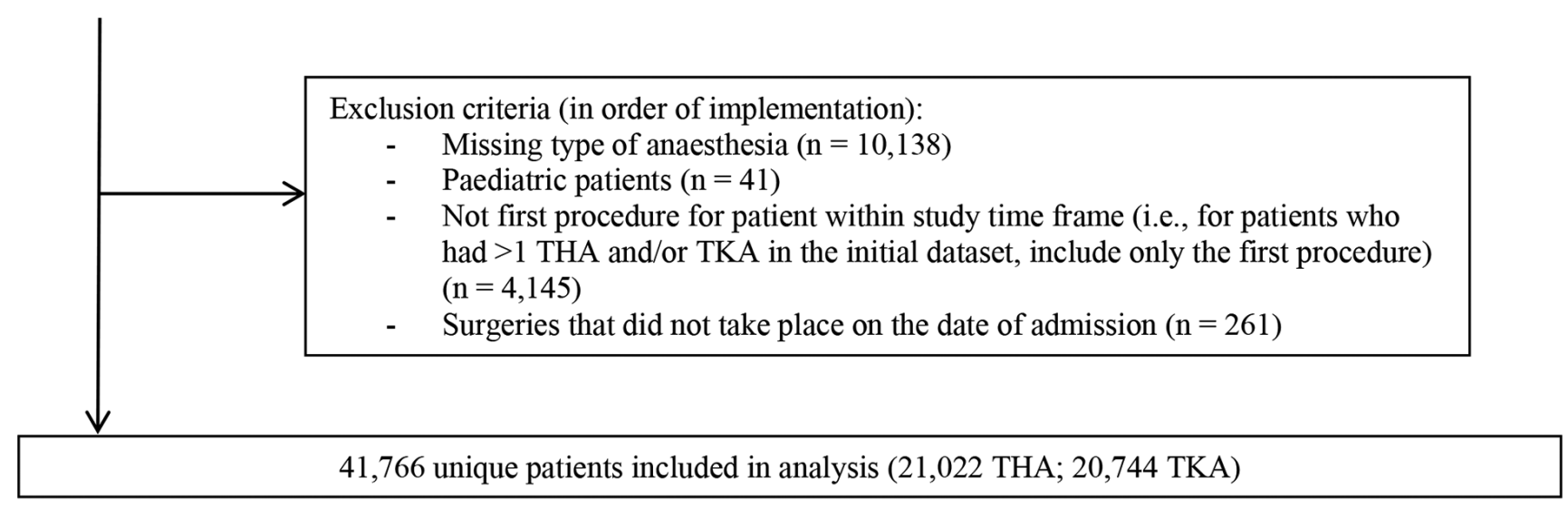

Figure 1 Summary of patient cohort and exclusion criteria ICD-9: International Classification of diseases, ninth revision THA: total hip arthroplasty TKA: total knee arthroplasty.

\section{Study variables}

The main effect of interest was OSA, which was defined as the presence of one or more of the following ICD-9 codes: 786.03, $780.53,780.51,780.57,327.2 \mathrm{X}$, or 278.03 .

The main outcomes (defined by ICD-9 codes) were cardiac, pulmonary, gastrointestinal, renal/genitourinary, and thromboembolic complications as well as delirium. Online supplementary appendix 1 contains a list of ICD-9 codes that were used to identify complications (Supplemental Digital Content 1). Additionally, prolonged LOS (characterized as exceeding the 75 th percentile of 4 days) was included as an outcome.

Categories for primary type of anesthesia were general or neuraxial; neuraxial anesthesia included combined spinal epidural, epidural, or spinal. Information on anesthesia type was extracted from a departmental anesthesia billing database.

Other study variables were age, sex, perioperative medications (benzodiazepines and ketamine), baseline laboratory values (hemoglobin, creatinine, international normalized ratio, and platelets), year of procedure (categorized every 2 years from 2005 to 2014), type of surgery (THA, TKA), and American Society of Anesthesiologists (ASA) physical status (I-II, III-IV). Individual comorbidities can be found in online supplementary appendix 2 (Supplemental Digital Content 2).

\section{Statistical analysis}

Descriptive summaries of patient characteristics were first considered. Categorical variables are presented as number (percent) and analyzed by OSA status using a $\chi^{2}$ test. Continuous variables are presented as median (IQR) and are analyzed using a t-test. Frequencies of missing data are also reported for both categorical and continuous variables as number (percent).

Using the statistical software $\mathrm{R}$ V.3.3.3 and RStudio V.1.0.136, ${ }^{13} 14$ we conducted multivariable logistic regression evaluating the impact of OSA on risk for the aforementioned outcomes.

All aforementioned study variables were included in the multivariable models except for obesity. A $\chi^{2}$ test of independence between the OSA and obesity comorbidity variables suggested a significant association between diagnoses of OSA and obesity in this patient population. In the interest of maintaining a parsimonious model (especially due to the relatively low frequencies of the outcomes considered), we included OSA but not obesity as a covariate in our models.

We used multiple imputation methods to estimate missing values using the R statistical package "mice" (multivariate imputation by chained equations). ${ }^{15}$ Frequencies of missing data are also reported for categorical and continuous variables as frequency (percent) (table 1). Results for multivariable logistic regression analyses were based on pooled estimates from five datasets generated from multiple imputation methods. Missing values were imputed using predictive mean matching (for continuous variables) and logistic regression imputation (for dichotomous variables).

The primary analysis was repeated as a sensitivity analysis in a subset of data that included only those patients with complete data (ie, excluding those with one or more missing values).

We report adjusted ORs and Bonferroni adjusted $\mathrm{p}$ values taking into account seven outcomes under study.

\section{RESULTS}

Of the 41766 unique patients (21 022 THA and 20744 TKA) included in this study, 7.7\% $(n=3228)$ had a diagnosis of OSA. OSA was more prevalent in patients with TKA than THA $(9.1 \%$ and $6.3 \%$, respectively; $\mathrm{p} \leq 0.001)$. The vast majority of surgeries $(97.8 \% ; n=40852)$ were performed under neuraxial anesthesia. We observed that patients with OSA had general anesthesia more often than patients without OSA $(3.1 \%$ and $2.1 \%$ general anesthesia, respectively; $\mathrm{p} \leq 0.001)$. Patients with OSA were younger, more likely to be men, classified with a higher ASA status, and had obesity more often than patients with no OSA (table 1). Univariable results also showed that patients with OSA had a higher incidence of pulmonary, gastrointestinal, and renal/genitourinary complications and prolonged LOS, but not delirium, cardiac, or thromboembolic complications.

Table 2 depicts results from the pooled multivariable logistic regression model after multiple imputation. After correcting for relevant covariates, OSA was significantly associated with an increased risk of pulmonary complications (OR 1.87, 95\% 
Table 1 Summary of baseline characteristics stratified by OSA status

\begin{tabular}{|c|c|c|c|}
\hline & No OSA (n=38 538) & OSA $(n=3228)$ & $P$ value \\
\hline Obesity & $7972(20.7)$ & $1501(46.5)$ & $<0.001$ \\
\hline \multicolumn{4}{|l|}{ ASA status } \\
\hline I-II & $35827(93.0)$ & $2427(75.2)$ & \multirow[t]{2}{*}{$<0.001$} \\
\hline III-IV & $2706(7.0)$ & $801(24.8)$ & \\
\hline Missing & $5(0.0)$ & $0(0.0)$ & \\
\hline \multicolumn{4}{|l|}{ Type of anesthesia } \\
\hline General & $814(2.1)$ & $100(3.1)$ & \multirow[t]{2}{*}{$<0.001$} \\
\hline Neuraxial & 37724 (97.9) & 3128 (96.9) & \\
\hline \multicolumn{4}{|l|}{ Time period } \\
\hline 2005-2006 & $2762(7.2)$ & $143(4.4)$ & \multirow[t]{5}{*}{$<0.001$} \\
\hline 2007-2008 & $7010(18.2)$ & $491(15.2)$ & \\
\hline 2009-2010 & $8388(21.8)$ & $704(21.8)$ & \\
\hline 2011-2012 & $9796(25.4)$ & $830(25.7)$ & \\
\hline $2013-2014$ & $10582(27.5)$ & $1060(32.8)$ & \\
\hline Age & $66.00(58.00-74.00)$ & $64.00(57.00-71.00)$ & $<0.001$ \\
\hline \multicolumn{4}{|l|}{ Gender } \\
\hline Female & $23427(60.8)$ & $1182(36.6)$ & \multirow[t]{2}{*}{$<0.001$} \\
\hline Male & $15111(39.2)$ & $2046(63.4)$ & \\
\hline \multicolumn{4}{|l|}{ Type of surgery } \\
\hline THA & $19690(51.1)$ & $1332(41.3)$ & \multirow[t]{2}{*}{$<0.001$} \\
\hline TKA & $18848(48.9)$ & $1896(58.7)$ & \\
\hline \multicolumn{4}{|l|}{ Baseline laboratory values } \\
\hline Hemoglobin & $13.30(12.40-14.30)$ & $13.70(12.70-14.60)$ & $<0.001$ \\
\hline Missing & $5526(14.3)$ & $464(14.4)$ & \\
\hline Creatinine & $0.90(0.70-1.00)$ & $0.90(0.80-1.10)$ & $<0.001$ \\
\hline Missing & $5613(14.6)$ & $470(14.6)$ & \\
\hline INR & $0.99(0.97-1.03)$ & $1.00(0.97-1.03)$ & $<0.001$ \\
\hline Missing & $5646(14.7)$ & $474(14.7)$ & \\
\hline Platelets & $251.00(212.00-297.00)$ & $241.00(201.00-284.00)$ & $<0.001$ \\
\hline Missing & $5537(14.4)$ & $465(14.4)$ & \\
\hline \multicolumn{4}{|l|}{ Perioperative benzodiazepines } \\
\hline No & $2038(5.3)$ & $152(4.7)$ & \multirow[t]{2}{*}{$<0.001$} \\
\hline Yes & $31170(80.9)$ & $2705(83.8)$ & \\
\hline Missing & $5330(13.8)$ & $371(11.5)$ & \\
\hline \multicolumn{4}{|l|}{ Perioperative ketamine } \\
\hline No & $25361(65.8)$ & $2139(66.3)$ & \multirow[t]{2}{*}{$<0.001$} \\
\hline Yes & $3634(9.4)$ & $476(14.7)$ & \\
\hline Missing & $9543(24.8)$ & $613(19.0)$ & \\
\hline \multicolumn{4}{|l|}{ Postoperative outcomes } \\
\hline Cardiac & $855(2.2)$ & $76(2.4)$ & 0.660 \\
\hline Pulmonary & $839(2.2)$ & $113(3.5)$ & $<0.001$ \\
\hline Gastrointestinal & $419(1.1)$ & $55(1.7)$ & 0.002 \\
\hline Renal/genitourinary & $869(2.3)$ & $94(2.9)$ & 0.020 \\
\hline Thromboembolic & $327(0.8)$ & $30(0.9)$ & 0.704 \\
\hline Delirium & $851(2.2)$ & $71(2.2)$ & 1.000 \\
\hline Prolonged LOS (>4 days) & $7344(19.1)$ & $782(24.2)$ & $<0.001$ \\
\hline
\end{tabular}

Categorical variables are summarized as frequency (\%) and are analyzed using $\chi^{2}$ test. Continuous variables are summarized as median (IQR) and are analyzed using t-test. Missing data are summarized as frequency (\%).

ASA, American Society of Anesthesiologists; INR, international normalized ratio; LOS, length of stay; OSA, obstructive sleep apnea; THA, total hip arthroplasty.

CI 1.51 to $2.30 ; \mathrm{p} \leq 0.007)$, gastrointestinal complications (OR $1.52,95 \%$ CI 1.13 to $2.04 ; \mathrm{p}=0.042$ ), and prolonged LOS (OR $1.44,95 \%$ CI 1.31 to $1.57 ; \mathrm{p} \leq 0.007)$. No significant associations were observed for the other outcomes.

Additionally, table 2 presents data on complications stratified by anesthesia type in the overall group of patients with OSA and without OSA. The use of neuraxial anesthesia was associated with decreased odds for pulmonary complications (OR 0.50, 95\% CI
0.36 to $0.69 ; \mathrm{p} \leq 0.007)$, renal/genitourinary complications (OR $0.50,95 \%$ CI 0.36 to $0.68 ; \mathrm{p} \leq 0.007$ ), delirium (OR $0.45,95 \%$ CI 0.32 to $0.62 ; \mathrm{p} \leq 0.007$ ), and prolonged LOS (OR $0.51,95 \%$ CI 0.44 to $0.59 ; \mathrm{p} \leq 0.007$ ), but not for cardiac, gastrointestinal, and thromboembolic complications.

Results from the sensitivity analysis excluding cases with missing information (cohort $\mathrm{n}=27$ 690) showed a similar pattern and confirmed findings from the imputed data: odds 
Table 2 Logistic regression models of postoperative outcomes by OSA status and anesthesia type after multiple imputation

\begin{tabular}{|c|c|c|c|c|}
\hline & \multicolumn{2}{|l|}{ OSA status } & \multicolumn{2}{|l|}{ Anesthesia type } \\
\hline & \multicolumn{2}{|l|}{ Yes (reference=no) } & \multicolumn{2}{|c|}{ Neuraxial $($ reference $=$ general) } \\
\hline & OR $(95 \% \mathrm{Cl})$ & $P$ value & OR $(95 \% \mathrm{Cl})$ & $P$ value \\
\hline Cardiac complications & 1.12 (0.88 to 1.43$)$ & $>0.999$ & $0.74(0.49$ to 1.10$)$ & 0.938 \\
\hline Pulmonary complications & 1.87 (1.51 to 2.30$)$ & $<0.007$ & $0.50(0.36$ to 0.69$)$ & $<0.007$ \\
\hline Gastrointestinal complications & 1.52 (1.13 to 2.04$)$ & 0.042 & $0.68(0.40$ to 1.17$)$ & $>0.999$ \\
\hline Renal/genitourinary complications & 1.19 (0.95 to 1.48$)$ & 0.931 & 0.50 (0.36 to 0.68$)$ & $<0.007$ \\
\hline Thromboembolic complications & 1.17 (0.79 to 1.72$)$ & $>0.999$ & 0.76 (0.39 to 1.50$)$ & $>0.999$ \\
\hline Delirium & 1.33 (1.03 to 1.72$)$ & 0.196 & 0.45 (0.32 to 0.63$)$ & $<0.007$ \\
\hline Prolonged LOS & 1.44 (1.31 to 1.57$)$ & $<0.007$ & 0.51 (0.44 to 0.59$)$ & $<0.007$ \\
\hline
\end{tabular}

Model adjusted for OSA status, type of surgery (TKA or THA), age at surgery (in years), time period in which the surgery occurred (categorized every 2 years from 2005 to 2014), sex (male or female), baseline laboratory values (hemoglobin, creatinine, INR, and platelets), primary type of anesthesia (neuraxial or general), dichotomized perioperative medications (benzodiazepines and ketamine), and ASA status (dichotomized as ASA status I-II or III-IV).

ASA, American Society of Anesthesiologists; INR, International normalized ratio; LOS, Length of stay; OSA, Obstructive sleep apnea; THA, Total hip arthroplasty; TKA, Total knee arthroplasty.

for pulmonary complications, gastrointestinal complications, and prolonged LOS were significantly increased in patients with OSA (OR 2.16, 95\% CI 1.69 to 2.73 , p $\leq 0.007$; OR $1.72,95 \%$ CI 1.19 to $2.42, \mathrm{p}=0.021$; and OR $1.57,95 \%$ CI 1.41 to 1.76 , $\mathrm{p} \leq 0.007$, respectively) (table 3 ).

\section{DISCUSSION}

In this retrospective cohort study using data from a high-volume specialty practice with near universal regional anesthesia use, we demonstrated that OSA was associated with an increased risk of pulmonary complications, gastrointestinal complications, and prolonged LOS in patients undergoing THA and TKA while no significant associations were seen for postoperative delirium, cardiac, renal/genitourinary, and thromboembolic complications.

Similar to other reports, we found that, while overall more women had lower joint arthroplasties, patients with OSA were more likely to be male. ${ }^{2}$ This finding may be explained by the fact that while female patients have a higher risk for osteoarthritis, ${ }^{16} 17$ male patients have higher rates of OSA. ${ }^{18} 19$

Furthermore, our data show that patients with OSA were more likely to have a higher ASA score. Indeed, OSA can be associated

Table 3 Logistic regression models of postoperative outcomes by OSA status for the dataset excluding patients with missing data

\begin{tabular}{|c|c|c|}
\hline & OSA status & \multirow[b]{3}{*}{$P$ value } \\
\hline & Yes (reference $=$ no) & \\
\hline & OR $(95 \% \mathrm{Cl})$ & \\
\hline Cardiac complications & 1.16 (0.85 to 1.55$)$ & $>0.999$ \\
\hline Pulmonary complications & 2.16 (1.69 to 2.73 ) & $<0.007$ \\
\hline Gastrointestinal complications & 1.72 (1.19 to 2.42$)$ & 0.021 \\
\hline Renal/genitourinary complications & 1.14 (0.86 to 1.49$)$ & $>0.999$ \\
\hline Thromboembolic complications & 1.11 (0.64 to 1.82$)$ & $>0.999$ \\
\hline Delirium & 1.46 (1.07 to 1.95$)$ & 0.091 \\
\hline Prolonged LOS & $1.57(1.41$ to 1.76$)$ & $<0.007$ \\
\hline
\end{tabular}

Model adjusted for type of surgery (TKA or THA), age at surgery (in years), time period in which the surgery occurred (categorized every 2 years from 2005 to 2014), sex (male or female), baseline laboratory values (hemoglobin, creatinine, INR, and platelets), primary type of anesthesia (neuraxial or general), dichotomized perioperative medications (benzodiazepines and ketamine), and ASA status (dichotomized as ASA status I-II or III-IV).

ASA, American Society of Anesthesiologists; INR, International normalized ratio; LOS, Length of stay; OSA, Obstructive sleep apnea; THA, Total hip arthroplasty; TKA, Total knee arthroplasty. with a variety of comorbidities. ${ }^{20}$ These include arterial hypertension, coronary heart disease, atrial fibrillation, stroke, and pulmonary hypertension. ${ }^{21-25}$

As found in previous studies, patients with OSA undergoing THA or TKA were younger than patients without OSA. ${ }^{2627}$ This could be related to the strong association between OSA and obesity, ${ }^{19} 28$ which is a predisposing factor for osteoarthritis of the lower extremity at a younger age. ${ }^{29} 30$

While both types of surgeries were performed with similar rates, patients with OSA were more prevalent in the TKA than the THA group. This too may be explained by the link between obesity and osteoarthritis of the knee. ${ }^{3132}$

\section{Pulmonary complications}

OSA was independently associated with an increase in risk of pulmonary complications. This has been described extensively in the literature. ${ }^{253}$ However, this is the first study investigating the impact of OSA on outcomes in a regional anesthesia setting, whereas in most other studies mainly general anesthesia was used. Theoretically, in a cohort of near universal neuraxial anesthesia use, minimal pulmonary risk differences between OSA groups are expected as neuraxial anesthesia is recommended by guidelines and has been shown to reduce perioperative pulmonary complications in patients with OSA. ${ }^{12}$ However, the increased risk found in our study may indicate that the diagnosis of OSA itself increases the risk of pulmonary complications even when general anesthesia is avoided. Interestingly, the OR found in the current study (1.87) for pulmonary complications was lower than those described by Kaw et al in a 2012 meta-analysis investigating OSA and postoperative outcome. They calculated total ORs for patients with OSA (compared with non-OSA) of 2.43, 2.05 , and 2.27 for the outcomes of respiratory failure, reintubation, and desaturation. ${ }^{5}$ As the predominant anesthesia type in these publications was general, this comparison may reflect a reduced risk of pulmonary complications for patients with OSA when regional anesthesia is used, but also shows that this risk cannot be completely eliminated by choice of anesthetic alone.

\section{Gastrointestinal complications}

We found that patients with OSA had an increased risk of developing gastrointestinal complications, which has not been widely described. Looking at the frequency of specific ICD-9 codes, we found that the most common diagnosis was paralytic ileus (560.1). 
Opioid consumption has been associated with the development of postoperative ileus. ${ }^{34}$ In our dataset, patients with OSA had general anesthesia more often than patients with no OSA and therefore most likely received higher doses of opioids. Although we corrected for type of anesthesia in our multivariable regression model, an effect related to this variable might still be present. Additionally, some studies suggest that sleep fragmentation, insomnia, and desaturation, which can be related to OSA, may be associated with increased sensitivity to pain. ${ }^{35-37}$ Patients with OSA may therefore require higher doses of opioids, which could directly influence the likelihood of opioid-related adverse effects like ileus. However, there are several publications that showed no difference or even a higher pain tolerance in patients with OSA. ${ }^{38-40}$

Another factor contributing to this finding may be the higher prevalence of irritable bowel syndrome among patients with OSA. Ghiasi et al found that OSA was independently associated with this condition. ${ }^{41}$ Chronic constipation, one of the key symptoms of irritable bowel syndrome, has been established as a risk factor for postoperative ileus. ${ }^{42}$

Obesity might also be a link between OSA and postoperative ileus. Obesity has been identified as a risk factor for postoperative ileus; however, this was observed mainly in patients undergoing abdominal surgery and its relevance for the orthopedic patient might be limited. ${ }^{43} 44$ There are investigations on postoperative ileus in joint arthroplasty patients, and Parvizi et al found that male gender, which is strongly associated with OSA, was a risk factor for postoperative ileus. ${ }^{45}$ In a 2001 retrospective cohort study investigating patients who underwent THA and TKA, Bederman et al concluded that male patients were at increased risk of having a more prolonged ileus. ${ }^{46}$

\section{Prolonged LOS}

In addition to the increased risk of developing complications, we showed that OSA had an impact on prolonged LOS. This is similar to findings from large retrospective cohort studies using national data, showing an increase in resource use in patients with OSA undergoing orthopedic surgery. ${ }^{26}{ }^{47}$ Prolonged LOS may at least in part be a consequence of increased morbidity, as patients with OSA may have longer hospital stays because of complications and resulting treatments or transfers to intensive care units. However, it could also reflect planned monitoring of patients with OSA and use of continuous positive airway pressure devices with the goal to reduce complications as recommended by the ASA task force on perioperative care of patients with OSA. ${ }^{7}$

\section{Limitations}

Our study is subject to a number of limitations. Because it is a retrospective study, we cannot determine causal relationships, but only associations. Additionally, our data were not collected for research, but for administrative purposes, which results in a lack of clinical detail. OSA status and complications are based on ICD-9 codes and may be subject to coding bias. Under-reporting of diagnoses may be present, especially for the diagnosis of OSA. Furthermore, this study included data from a single institution and generalizability to a broader surgical population might be limited.

\section{CONCLUSIONS}

In conclusion, we showed that in a regional anesthesia setting, OSA was associated with an increased risk of prolonged LOS, and pulmonary and gastrointestinal complications, but not for delirium, thromboembolism, cardiac, and renal/genitourinary complications. This underlines that, while regional anesthesia may improve postoperative outcomes in patients with OSA, some complications cannot be eliminated.

Future studies should try and investigate if among neuraxial techniques one has a better potential to reduce the risk of complications in patients with OSA than other neuraxial techniques. Furthermore, as we know that patients with OSA undergoing lower joint arthroplasty are at risk even in a regional anesthesia setting, other interventions that could potentially lead to a better outcome in this patient group should be investigated.

\section{Patient consent Not required.}

Ethics approval This study was approved by our Institutional Review Board (IRB no. 2016-436) and considered exempt from obtaining patient consent as a retrospective study.

\section{REFERENCES}

1 Chung F, Ward B, Ho J, et al. Preoperative identification of sleep apnea risk in elective surgical patients, using the Berlin questionnaire. J Clin Anesth 2007;19:130-4.

2 Memtsoudis S, Liu SS, Ma Y, et al. Perioperative pulmonary outcomes in patients with sleep apnea after noncardiac surgery. Anesth Analg 2011;112:113-21.

3 Peppard PE, Young T, Barnet JH, et al. Increased prevalence of sleep-disordered breathing in adults. Am J Epidemio/ 2013;177:1006-14.

4 Hai F, Porhomayon J, Vermont L, et al. Postoperative complications in patients with obstructive sleep apnea: a meta-analysis. J Clin Anesth 2014;26:591-600.

5 Kaw R, Chung F, Pasupuleti V, et al. Meta-analysis of the association between obstructive sleep apnoea and postoperative outcome. Br J Anaesth 2012;109:897-906.

6 Mokhlesi B, Hovda MD, Vekhter B, et al. Sleep-disordered breathing and postoperative outcomes after elective surgery: analysis of the nationwide inpatient sample. Chest 2013;144:903-14.

7 American Society of Anesthesiologists Task Force on Perioperative Management of patients with obstructive sleep apnea. Practice guidelines for the perioperative management of patients with obstructive sleep apnea: an updated report by the American Society of Anesthesiologists Task Force on Perioperative Management of patients with obstructive sleep apnea. Anesthesiology 2014;120:268-86.

8 Chung F, Memtsoudis SG, Ramachandran SK, et al. Society of Anesthesia and Sleep Medicine guidelines on preoperative screening and assessment of adult patients with obstructive sleep apnea. Anesth Analg 2016;123:452-73.

9 Joshi GP, Ankichetty SP, Gan TJ, et al. Society for Ambulatory Anesthesia consensus statement on preoperative selection of adult patients with obstructive sleep apnea scheduled for ambulatory surgery. Anesth Analg 2012;115:1060-8.

10 Memtsoudis SG, Stundner O, Rasul R, et al. Sleep apnea and total joint arthroplasty under various types of anesthesia: a population-based study of perioperative outcomes. Reg Anesth Pain Med 2013;38:274-81.

11 Ambrosii T, Şandru S, Belîi A. The prevalence of perioperative complications in patients with and without obstructive sleep apnoea: a prospective cohort study. Rom J Anaesth Intensive Care 2016;23:103-10.

12 Naqvi SY, Rabiei AH, Maltenfort MG, et al. Perioperative complications in patients with sleep apnea undergoing total joint arthroplasty. J Arthroplasty 2017;32:2680-3.

13 R core team. R: Language and environment for statistical computing 3.3.3 edn. Vienna, Austria: R Foundation for Statistical Computing, 2017.

14 Rstudio team. Rstudio: Integrated development for R. 1.0.136 edn. Boston, MA: Rstudio, inc, 2016.

15 Sv B, Groothuis-Oudshoorn K. Mice: multivariate imputation by chained equations in R. J Stat Softw 2010:1-68.

16 Maillefert JF, Gueguen A, Monreal M, et al. Sex differences in hip osteoarthritis: results of a longitudinal study in 508 patients. Ann Rheum Dis 2003;62:931-4.

17 Davis MA, Ettinger WH, Neuhaus JM, et al. Sex differences in osteoarthritis of the knee. The role of obesity. Am J Epidemiol 1988;127:1019-30.

18 Tufik S, Santos-Silva R, Taddei JA, et al. Obstructive sleep apnea syndrome in the Sao Paulo Epidemiologic Sleep Study. Sleep Med 2010;11:441-6.

19 Young T, Skatrud J, Peppard PE. Risk factors for obstructive sleep apnea in adults. JAMA 2004;291:2013-6.

20 Tveit RL, Lehmann S, Bjorvatn B. Prevalence of several somatic diseases depends on the presence and severity of obstructive sleep apnea. PLoS One 2018;13:e0192671.

21 Peppard PE, Young T, Palta M, et al. Prospective study of the association between sleep-disordered breathing and hypertension. N Engl J Med 2000;342:1378-84.

22 Punjabi NM, Caffo BS, Goodwin JL, et al. Sleep-disordered breathing and mortality: a prospective cohort study. PLoS Med 2009;6:e1000132.

23 Gottlieb DJ, Yenokyan G, Newman AB, et al. Prospective study of obstructive sleep apnea and incident coronary heart disease and heart failure: the sleep heart health study. Circulation 2010;122:352-60. 
24 Redline S, Yenokyan G, Gottlieb DJ, et al. Obstructive sleep apnea-hypopnea and incident stroke: the sleep heart health study. Am J Respir Crit Care Med 2010;182:269-77.

25 Cadby G, McArdle N, Briffa T, et al. Severity of OSA is an independent predictor of incident atrial fibrillation hospitalization in a large sleep-clinic cohort. Chest 2015;148:945-52.

26 Memtsoudis SG, Stundner 0, Rasul R, et al. The impact of sleep apnea on postoperative utilization of resources and adverse outcomes. Anesth Analg 2014;118:407-18.

27 Cozowicz C, Poeran J, Olson A, et al. Trends in perioperative practice and resource utilization in patients with obstructive sleep apnea undergoing joint arthroplasty. Anesth Analg 2017;125:66-77

28 Peppard PE, Young T, Palta M, et al. Longitudinal study of moderate weight change and sleep-disordered breathing. JAMA 2000;284:3015-21.

29 Heliövaara M, Mäkelä M, Impivaara O, et al. Association of overweight, trauma and workload with coxarthrosis. A health survey of 7,217 persons. Acta Orthop Scand 1993;64:513-8.

30 Felson DT, Anderson JJ, Naimark A, et al. Obesity and knee osteoarthritis. The Framingham Study. Ann Intern Med 1988;109:18-24.

31 Gelber AC, Hochberg MC, Mead LA, et al. Body mass index in young men and the risk of subsequent knee and hip osteoarthritis. Am J Med 1999;107:542-8.

32 Hartz AJ, Fischer ME, Bril G, et al. The association of obesity with joint pain and osteoarthritis in the HANES data. J Chronic Dis 1986;39:311-9.

33 Opperer M, Cozowicz C, Bugada D, et al. Does obstructive sleep apnea influence perioperative outcome? A qualitative systematic review for the Society of Anesthesia and Sleep Medicine Task Force on preoperative preparation of patients with sleepdisordered breathing. Anesth Analg 2016;122:1321-34.

34 Gan TJ, Robinson SB, Oderda GM, et al. Impact of postsurgical opioid use and ileus on economic outcomes in gastrointestinal surgeries. Curr Med Res Opin 2015;31:677-86.

35 Roehrs T, Hyde M, Blaisdell B, et al. Sleep loss and REM sleep loss are hyperalgesic. Sleep 2006;29:145-51.
36 Haack M, Scott-Sutherland J, Santangelo G, et al. Pain sensitivity and modulation in primary insomnia. Eur J Pain 2012;16:522-33.

37 Doufas AG, Tian L, Davies MF, et al. Nocturnal intermittent hypoxia is independently associated with pain in subjects suffering from sleep-disordered breathing. Anesthesiology 2013;119:1149-62.

38 Doufas AG, Tian L, Padrez KA, et al. Experimental pain and opioid analgesia in volunteers at high risk for obstructive sleep apnea. PLoS One 2013:8:e54807.

39 Turan A, You J, Egan C, et al. Chronic intermittent hypoxia is independently associated with reduced postoperative opioid consumption in bariatric patients suffering from sleep-disordered breathing. PLoS One 2015;10:e0127809.

40 Smith MT, Wickwire EM, Grace EG, et al. Sleep disorders and their association with laboratory pain sensitivity in temporomandibular joint disorder. Sleep 2009:32:779-90

41 Ghiasi F, Amra B, Sebghatollahi V, et al. Association of irritable bowel syndrome and sleep apnea in patients referred to sleep laboratory. J Res Med Sci 2017;22:72

42 Lee TH, Lee JS, Hong SJ, et al. Risk factors for postoperative ileus following orthopedic surgery: the role of chronic constipation. J Neurogastroenterol Motil 2015:21:121-5

43 Svatek RS, Fisher MB, Williams MB, et al. Age and body mass index are independent risk factors for the development of postoperative paralytic ileus after radical cystectomy. Urology 2010;76:1419-24.

44 Murphy MM, Tevis SE, Kennedy GD. Independent risk factors for prolonged postoperative ileus development. J Surg Res 2016;201:279-85.

45 Parvizi J, Han SB, Tarity TD, et al. Postoperative ileus after total joint arthroplasty. $J$ Arthroplasty 2008;23:360-5

46 Bederman SS, Betsy M, Winiarsky R, et al. Postoperative ileus in the lower extremity arthroplasty patient. J Arthroplasty 2001;16:1066-70.

47 Stundner O, Chiu YL, Sun X, et al. Sleep apnoea adversely affects the outcome in patients who undergo posterior lumbar fusion: a population-based study. Bone Joint $J$ 2014;96-B:242-8. 